Article

\title{
Tooth Discoloration after Regenerative Endodontic Procedures with Calcium Silicate-Based Cements-An Ex Vivo Study
}

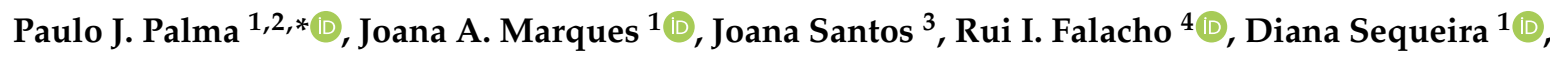 \\ Patrícia Diogo ${ }^{1,2}$, Francisco Caramelo ${ }^{5}\left(\mathbb{C}\right.$, João C. Ramos ${ }^{2,6}$ and João Miguel Santos ${ }^{1,2}$ (I) \\ 1 Institute of Endodontics, Faculty of Medicine, University of Coimbra, 3000-075 Coimbra, Portugal; \\ joanaamarques@uc.pt (J.A.M.); dianasequeira@fmed.uc.pt (D.S.); pdn@uc.pt (P.D.); \\ jsantos@fmed.uc.pt (J.M.S.) \\ 2 Center for Innovation and Research in Oral Sciences (CIROS), Faculty of Medicine, University of Coimbra, \\ 3000-075 Coimbra, Portugal; jcramos@fmed.uc.pt \\ 3 Dentistry Department, Faculty of Medicine, University of Coimbra, 3000-075 Coimbra, Portugal; \\ uc2014207604@student.uc.pt \\ 4 Institute of Oral Implantology and Prosthodontics, Faculty of Medicine, University of Coimbra, \\ 3000-075 Coimbra, Portugal; rifalacho@fmed.uc.pt \\ 5 Coimbra Institute for Clinical and Biomedical Research (iCBR) and Laboratory of Biostatistics and Medical \\ Informatics (LBIM), Faculty of Medicine, University of Coimbra, 3000-548 Coimbra, Portugal; \\ fcaramelo@fmed.uc.pt \\ 6 Institute of Operative Dentistry, Faculty of Medicine, University of Coimbra, 3000-075 Coimbra, Portugal \\ * Correspondence: ppalma@uc.pt; Tel.: +351-239-249-151
}

Received: 13 July 2020; Accepted: 19 August 2020; Published: 21 August 2020

check for updates

\begin{abstract}
The aim of the present ex vivo study was to assess and compare coronal discoloration induced by four endodontic biomaterials used in regenerative endodontic procedures (REPs). Root resection was executed horizontally, $2 \mathrm{~mm}$ apical to the cementoenamel junction, in all fifty-four teeth. After accessing the pulp chamber, specimens were randomly divided in groups and filled with either saline solution or blood, followed by calcium silicate-based cements (CSCs) placement: ProRoot mineral trioxide aggregate (MTA) (Dentsply Sirona), Biodentine (Septodont), TotalFill BC (FKG), or pulp capping material (PCM) (Coltène). Color change $(\triangle E)$ was assessed using the $L^{*} a^{*}$ $\mathrm{b}^{*}$ system at five different timepoints (before and immediately after biomaterial application, $72 \mathrm{~h}$, 7 days, and 6 months). The significance level for statistical analysis was set at $p<0.05$. There are statistically significant differences regarding $\Delta \mathrm{E}$ over time $(p<0.001)$. Statistical differences are found considering material $(p<0.001)$, treatment $(p=0.007)$, or both $(p=0.002)$. If solely the material or treatment is considered, regardless of time, statistically significant differences are detected $(p<0.001)$. After a six-month period of evaluation, blood exposure might be a critical factor in biomaterials' color variation. Biodentine presents the lowest discoloration potential, followed by TotalFill and PCM, albeit without statistically significant differences. MTA exhibited the greatest color variation. The selection of biomaterial should consider the material's discoloration potential.
\end{abstract}

Keywords: calcium silicate-based cements; endodontic biomaterials; regenerative endodontic procedures; tooth discoloration; vital pulp therapy

\section{Introduction}

Regenerative endodontic procedures (REPs) demonstrate excellent success rates for the resolution of periapical pathology and increase survival of the immature tooth [1-3]. However, tooth discoloration 
is a major esthetic concern reported in multiple studies as an undesirable result of regenerative endodontic procedures [4-6] or vital pulp therapy [7]. REPs are defined as "biologically based procedures designed to replace damaged tooth structures, including dentine and root structures, as well as cells of the pulp-dentine complex" [8]. Therefore, REPs aim is to regenerate the pulp-dentine complex damaged in immature permanent teeth with pulp necrosis [9]. The association between color variation and the use of different endodontic materials in REPs, namely calcium silicate-based cements (CSCs) such as mineral trioxide aggregate-MTA (ProRoot MTA, Dentsply Sirona, Johnson City, TN, USA) — and Biodentine (Septodont, Saint-Maur-des-Fossés, France), has been described [10,11]. Thus, the selection of the biomaterial must consider functional, biological, and esthetic aspects, including its discoloration potential [12-14].

MTA was developed as a filling material, presenting a wide span of clinical applications including REPs, pulp capping procedures, apexification and apexogenesis, as well as root resorptions, furcation defects, and perforation repairs [15,16]. Although this bioceramic shows excellent biocompatibility and bioactivity, the original formulation (gray MTA) was associated with coronal discoloration [16]. This well-known drawback triggered the development of white MTA, by modifying the originally marketed composition. However, the latter formulation still results in dental staining $[11,14,17,18]$. Considering MTA's main disadvantages, including its discoloration potential, a new generation of CSCs was introduced, such as Biodentine (with zirconium oxide as radiopacifier), pre-mixed TotalFill BC RRM Putty (FKG, La Chaux-de-Fonds, Switzerland) and an under development experimental material (pulp capping material (PCM), Coltène/Whaledent, Altstätten, Switzerland).

Nowadays, multiple hypotheses have been outlined regarding the underlying mechanisms of color alteration following REPs, with the presence of bismuth oxide within the material composition as radiopacifier [19-21] and blood contamination [22,23] being suggested as key factors to trigger and exacerbate discoloration, respectively.

Current literature states that Biodentine presents superior color stability over MTA, which might be associated with the faster setting time of the formerly mentioned biomaterial $[11,18,24]$. Therefore, bearing this potential explanation in mind, this study aims to analyze the discoloration potential of two more recently presented calcium silicate-based cements-TotalFill BC Putty and a new experimental cement (PCM) - which were selected because of their shorter setting times (approximately $2 \mathrm{~h}$ and $3 \mathrm{~min}$, respectively) compared to MTA. Moreover, the present study belongs to an ongoing research line $[3,11]$ that aims to investigate and compare the discoloration potential of several available bioactive cements used in regenerative therapy. Since the last study [24] of the mentioned research project was conducted on acrylic teeth and evaluated the role played by blood on color stability of both Biodentine and MTA, the present study intends to confirm the possible interaction between different biomaterials and blood, when in contact with the human tooth dental structure.

Thus, the aim of the present ex vivo study was to assess and compare coronal discoloration induced by four endodontic biomaterials used in REPs-ProRoot MTA, Biodentine, TotalFill BC Putty, and an experimental pulp capping material (PCM) - in the presence of saline solution or blood. The null hypothesis states that there are no statistically significant differences between the experimental groups.

\section{Experimental Section}

\subsection{Specimen Preparation}

Fifty-four premolars extracted for orthodontic purposes or periodontal reasons were included in the present study. The number of samples included in the present study was based on a previous sample size calculation, performed in $G^{*}$ Power 3.1 software. Only teeth clinically and radiographically free of caries, cracks, restorations, and pathologic or extrinsic discolorations were selected for the experimental procedures. External surfaces of each tooth were visually inspected and cleaned with ultrasonic scaler and periodontal scalers, and polished with pumice and water in order to remove any organic material, calculus, or extrinsic staining. 
Root resection was executed horizontally, $2 \mathrm{~mm}$ apical to the cementoenamel junction, in all teeth.

The access cavity was prepared in all specimens through root-end preparation using a cylindrical diamond bur (2-mm diameter, Drendel+Zweiling Diamant GmbH, Kalletal, Germany) with high speed turbine, under copious irrigation. Cavities centered on the pulp chamber with $4 \mathrm{~mm}$ depth and $2 \mathrm{~mm}$ diameter were obtained, always ensuring a peripheral minimum of $1 \mathrm{~mm}$ of enamel and $1 \mathrm{~mm}$ of dentin. The access cavities were then irrigated with sodium hypochlorite $(2.5 \% \mathrm{NaOCl})$ to remove pulp tissue remnants, followed by a 17\% EDTA (CanalPro EDTA, Coltène/Whaledent, Altstätten, Switzerland) irrigation to eliminate the smear layer and expose tubular dentin, and then a final rinse with saline solution $(0.9 \% \mathrm{NaCl})$.

Specimens were stored in saline solution until experimental procedures started.

\subsection{Blood Collection}

Informed consent was obtained from a participant, according to the approval of the Ethical Committee of IRB of the Faculty of Medicine-University of Coimbra (notification CE001/2013), and a blood $(6 \mathrm{~mL})$ sample was collected by venipuncture. The blood collection tubes were sterile and internally coated with spray-dried tripotassium ethylenediaminetetraacetic acid (K3EDTA) to prevent clotting, thus allowing both hematology analysis and handling during sample preparation. Hematologic parameters included hematocrit and hemoglobin determinations and an erythrocyte count. The obtained values were $37.2 \%$ for hematocrit, $4.22 \times 10^{12} / \mathrm{L}$ for erythrocytes, and $11.9 \mathrm{~g} / \mathrm{dL}$ for hemoglobin. Blood samples were sealed and stored at $4{ }^{\circ} \mathrm{C}$ until use.

\subsection{Experimental Setup}

Samples were randomly divided into 10 groups by the stratified random sampling method: one negative control group $(n=3)$, one positive control group $(n=3)$, and 8 experimental groups $(n=48)$, as shown in Figure 1.

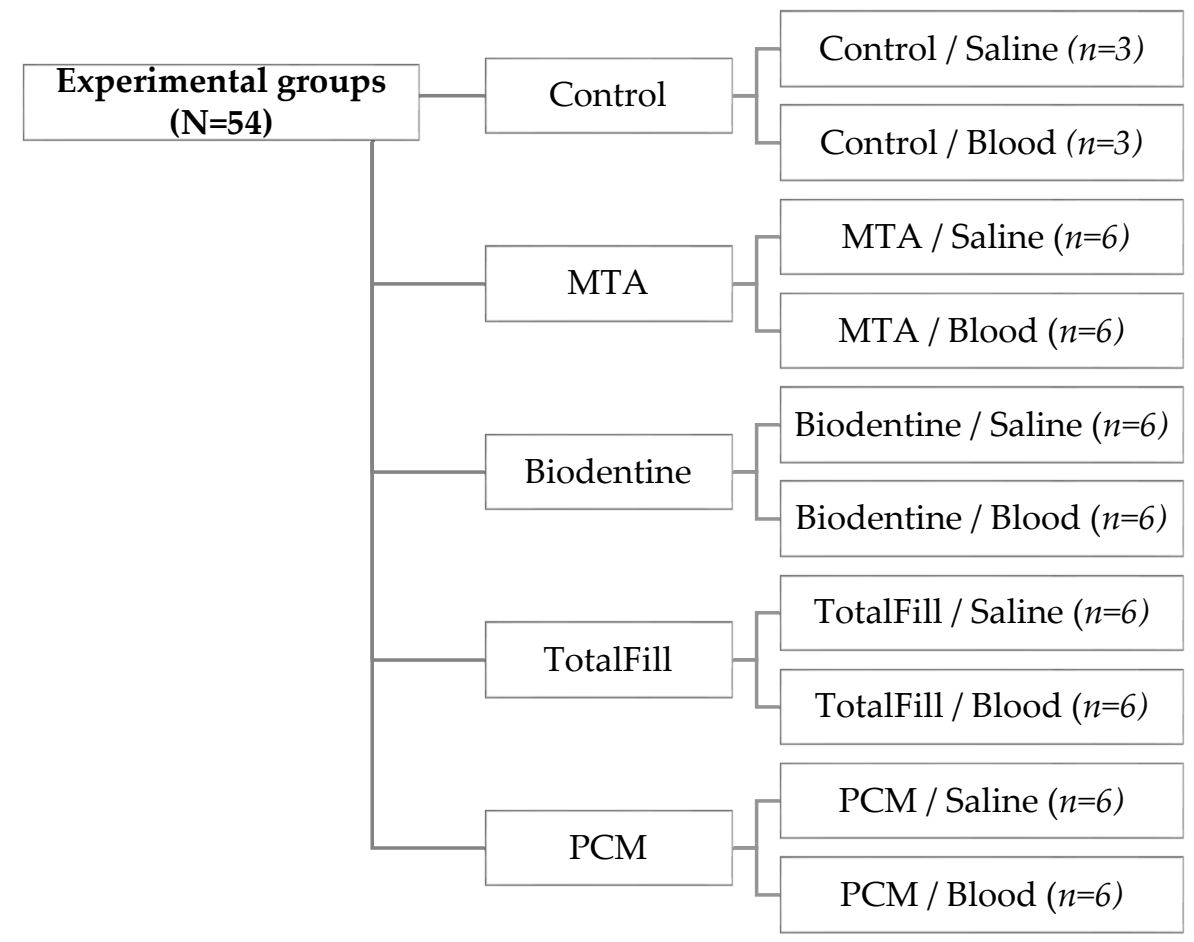

Figure 1. Experimental groups description (Saline: saline solution; MTA: ProRoot MTA; TotalFill: TotalFill BC Putty; PCM: experimental pulp capping material). 
After preoperative color measurement of each specimen, cavities in the control group were filled with a sterile cotton pellet moistened with saline solution (negative control-Control/Saline) and blood (positive control-Control/Blood), respectively. The access cavities of the remaining experimental groups were filled with different combinations of liquid solution (saline solution or blood) and biomaterial (ProRoot MTA, Biodentine, TotalFill BC, and PCM) as follows: MTA/Saline; MTA/Blood; Biodentine/Saline; Biodentine/Blood; TotalFill/Saline; TotalFill/Blood; PCM/Saline and PCM/Blood.

Cavities were firstly filled with either saline solution or blood, using a syringe and needle with lateral exit (Kendall, Monoject, New York, NY, USA). Subsequently, biomaterials were prepared according to the manufacturers' instructions (Table 1) and inserted into the cavities directly over the liquid solutions. All cavities were then sealed with a resin-based flowable composite-SDR Flow+ Bulk Fill Flowable A2 (Dentsply DeTrey GmbH, Konstanz, Germany)—, and light-cured for $20 \mathrm{~s}$ with a polywave LED curing light source (Bluephase Style, Ivoclar Vivadent AG, Schaan, Liechtenstein).

Table 1. Materials' compositions, manufacturers, preparation procedures, lot numbers and expiration dates.

\begin{tabular}{|c|c|c|c|c|c|}
\hline Material & Composition & Manufacturer & Preparation Procedure & Lot Number & $\begin{array}{l}\text { Expiration } \\
\text { Date }\end{array}$ \\
\hline ProRoot ${ }^{\circledR}$ MTA & $\begin{array}{l}\text { Tricalcium silicate, bismuth oxide, } \\
\text { dicalcium silicate, tricalcium aluminate, } \\
\text { calcium sulfate dehydrates or gypsum }\end{array}$ & $\begin{array}{l}\text { Dentsply Sirona, Johnson } \\
\text { City, TN, USA }\end{array}$ & Mix powder/liquid ratio 1:3 & 177918 & $08 / 2020$ \\
\hline Biodentine $^{\mathrm{TM}}$ & $\begin{array}{l}\text { Powder: tricalcium silicate, dicalcium } \\
\text { silicate, calcium carbonate and oxide, } \\
\text { iron oxide, zirconium oxide } \\
\text { Liquid: calcium chloride, } \\
\text { hydrosoluble polymer }\end{array}$ & $\begin{array}{l}\text { Septodont, } \\
\text { Saint-Maur-des-Fossés, } \\
\text { France }\end{array}$ & $\begin{array}{l}\text { Pour } 5 \text { drops of liquid into } \\
\text { the capsule } \\
\text { Place the capsule on a } \\
\text { mixing device } \\
\text { Mix for } 30 \mathrm{~s}\end{array}$ & B21190 & $11 / 2019$ \\
\hline $\begin{array}{l}\text { TotalFill }^{\circledR} \text { BC } \\
\text { RRM }^{\mathrm{TM}} \text { Putty }\end{array}$ & $\begin{array}{l}\text { Tricalcium silicate, tantalum oxide, } \\
\text { zirconium oxide }\end{array}$ & $\begin{array}{l}\text { FKG, La Chaux-de-Fonds, } \\
\text { Switzerland }\end{array}$ & $\begin{array}{l}\text { No mixing is required } \\
\text { Remove the material and } \\
\text { place it on glass slab. } \\
\text { Place the material into canal } \\
\text { with an instrument and } \\
\text { compress it. }\end{array}$ & 1702BPP & $11 / 2019$ \\
\hline PCM & $\begin{array}{l}\text { Silicates, polydimethylsiloxane, silicon } \\
\text { oils, platinum catalyst, zinc oxide, } \\
\text { zirconium dioxide, bioactive } \\
\text { glass, pigment }\end{array}$ & $\begin{array}{l}\text { Coltène/Whaledent, } \\
\text { Altstätten, Switzerland }\end{array}$ & $\begin{array}{l}\text { Ready to apply using } \\
\text { auto-mixing tips }\end{array}$ & 2018120-P3-RR & 08/2019 \\
\hline $\begin{array}{l}\mathrm{SDR}^{\mathrm{TM}} \text { Bulk fill } \\
\text { flowable } \\
\text { composite }\end{array}$ & $\begin{array}{l}\text { Barium-alumino-fluoro-borosilicate } \\
\text { glass, strontium-alumino-fluoro-silicate } \\
\text { glass, modified } \\
\text { urethanedimethacrylateresin, } \\
\text { EBPADMA, TEGMA, CQ, } \\
\text { photoaccelerator, BHT, UV stabilizer, } \\
\text { titanium dioxide, iron oxide pigments, } \\
\text { fluorescing agent }\end{array}$ & $\begin{array}{l}\text { Dentsply DeTrey GmbH, } \\
\text { Konstanz, Germany }\end{array}$ & $\begin{array}{l}\text { Dispense SDRTM material } \\
\text { Light-cure for at least } 20 \mathrm{~s}\end{array}$ & 1803000656 & $02 / 2021$ \\
\hline
\end{tabular}

Tooth filling and photographic record were performed in the laboratory at $23.5{ }^{\circ} \mathrm{C}$ in a $52 \%$ humidity environment. Immediate postoperative color measurements were recorded, and specimens were stored in a dark environment, in an incubator (Gallenkamp, London, UK) at $37{ }^{\circ} \mathrm{C}$ and $100 \%$ humidity until subsequent color measurement evaluation periods.

\subsection{Photographic Record}

Photographic register was performed with a Canon EOS 5DsR camera using a Canon EF $100 \mathrm{~mm}$ f/2.8 L Macro IS USM Lens and a Canon Macro Twin Lite MT-24EX with emitters positioned at a $45^{\circ}$ angle and cross polarization filters (polar_eyes, Emulation Group). The following settings were used: F22 aperture, ISO 100, 1/125 shutter speed, Flash at Manual $\frac{1}{2}$ power and custom white balance (with a $18 \%$ grey card-eLAB-Emulation Group). The photographs were taken from the buccal wall/side of the teeth and were saved in RAW file format.

In order to assure standard positioning of the samples, a silicone device (Virtual Refill Putty Fast Set, Ivoclar Vivadent AG, Schaan, Liechtenstein) was prepared to hold the specimens and the grey card scale was used as a reference to properly center and place the samples, as seen in Figure 2. 


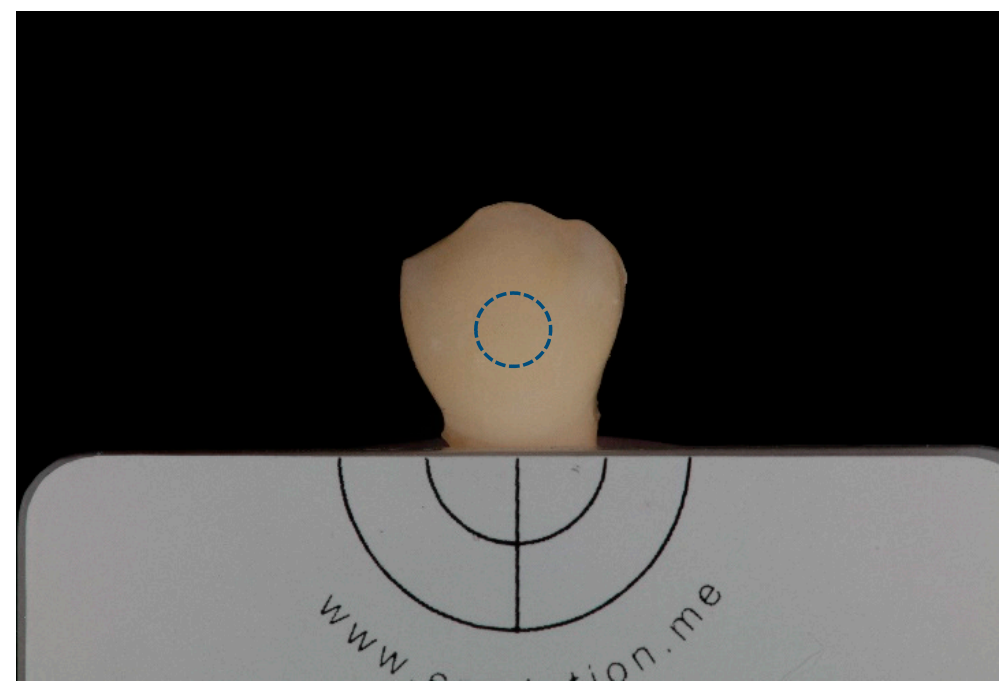

Figure 2. Positioning device for photographic record. The blue dashed line delimits the total area considered for shade analysis within the Commission International de l'Eclairage (CIE) $\mathrm{L}^{*} \mathrm{a}^{*} \mathrm{~b}^{*}$ color space for all timeframes.

In post-production, before taking color measurements, all photographic registers were calibrated using the White Balance Selector and the Exposure slider of Adobe Photoshop Lightroom (Adobe Systems, San Jose, CA, USA).

\subsection{Tooth Color Measurement}

In shade analysis there are five evaluation periods (Figure 3): $\mathrm{T}_{0}$ : baseline (after cavity preparation, before biomaterial placement); $\mathrm{T}_{\mathrm{PO}}$ : immediately after biomaterial placement and provisional restoration; $\mathrm{T}_{72 \mathrm{H}}$ : after $72 \mathrm{~h}$ of storage; $\mathrm{T}_{7 \mathrm{D}}$ : after 7 days of storage; and $\mathrm{T}_{6 \mathrm{M}}$ : after six months of storage.

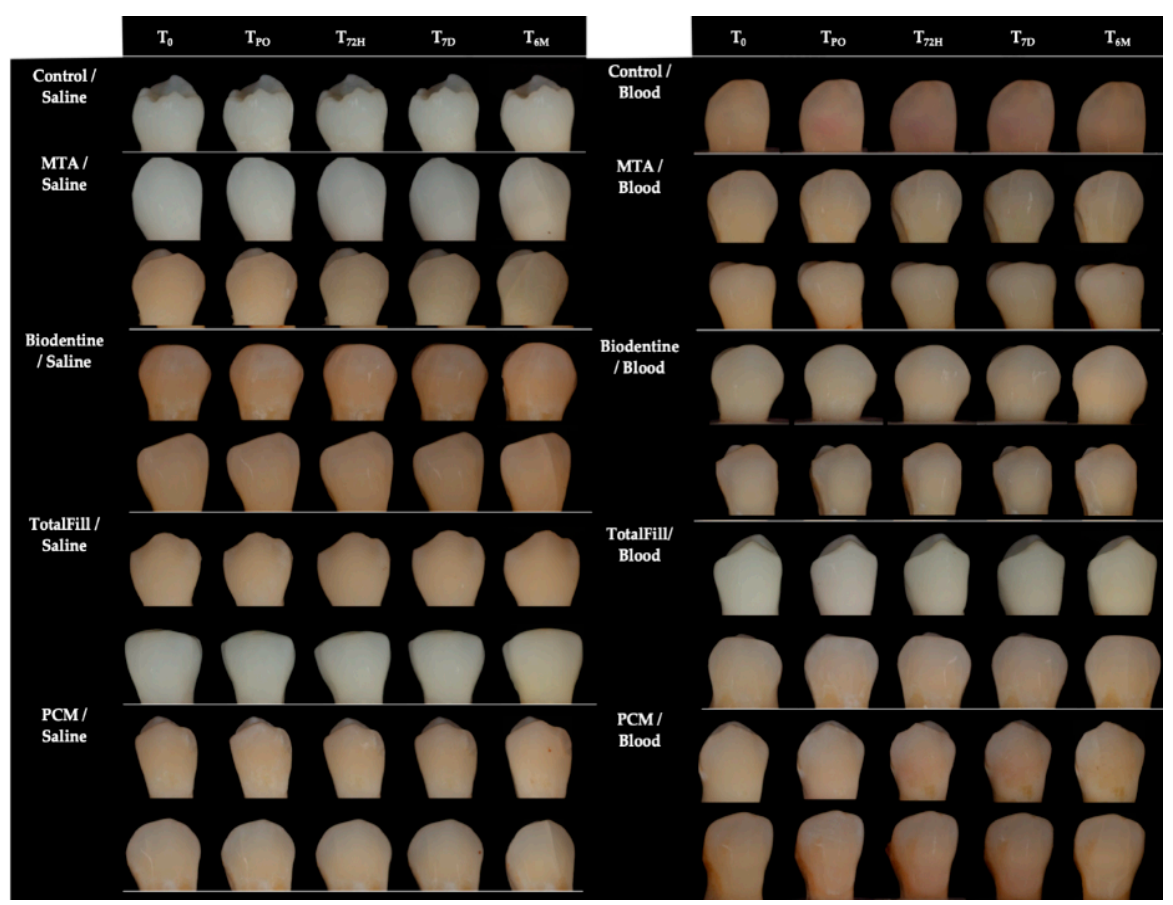

Figure 3. Color assessment of randomly selected representative samples from each experimental group at the five evaluated timeframes $\left(\mathrm{T}_{0}, \mathrm{~T}_{\mathrm{PO}}, \mathrm{T}_{72 \mathrm{H}}, \mathrm{T}_{7 \mathrm{D}}\right.$, and $\left.\mathrm{T}_{6 \mathrm{M}}\right)$. 
Color assessment was performed by a single operator using ImageJ (National Institutes of Health, NIH) software, considering a central circular area, focusing on the height and width of the tooth crown, with the total area of $256 \times 256$ pixels. The color space was measured with the rules of the Commission International de l'Eclairage (CIE) $\mathrm{L}^{*} \mathrm{a}^{*} \mathrm{~b}^{*}$ system, with $\mathrm{L}^{*}$ values corresponding to the lightness or luminance (ranging from 0 [black] to 100 [white]), $\mathrm{a}^{*}$ values matching the red-green axis (red-positive $a^{*}$; green-negative $a^{*}$ ) and the values of $b^{*}$ exhibiting the yellow-blue axis (yellow-positive $b^{*}$; blue-negative $b^{*}$ ). $\Delta E$ describes the color variation between baseline and each of the subsequent color measurement periods of evaluation $\left(\mathrm{T}_{\mathrm{PO}}, \mathrm{T}_{72 \mathrm{H}}, \mathrm{T}_{7 \mathrm{D}}\right.$, and $\left.\mathrm{T}_{6 \mathrm{M}}\right)$, determined by using the following formula:

$$
\Delta \mathrm{E}=\left[(\Delta \mathrm{L})^{2}+(\Delta \mathrm{a})^{2}+(\Delta \mathrm{b})^{2}\right]^{1 / 2}
$$

Perceptible chromatic alterations for the human eye were defined at the threshold value of $\Delta \mathrm{E} \geq 3.3$ [25].

\subsection{Statistical Analysis}

Statistical analysis was performed using IBMSPSSStatistics version 24 software and the significance level was set at $\alpha=0.05$. The description of the results regarding each material (MTA, Biodentine, TotalFill, and PCM) and each treatment option (saline solution or blood) was attained using the mean and standard deviation. To evaluate color variation over time, considering both the biomaterial and the treatment, a repeated measures ANOVA with two independent factors was applied. For each material and condition, a graph was created depicting the chromatic coordinates $\left(\mathrm{L}^{*}, \mathrm{a}^{*}, \mathrm{~b}^{*}\right)$ and the variation of $\Delta \mathrm{E}$ over time. A Friedman test allowed assessment of the differences over time. Kruskal-Wallis testing was executed to evaluate the differences between the groups at $\mathrm{T}_{6 \mathrm{M}}$. Kruskal-Wallis testing was repeated for each condition.

\section{Results}

Table 2 shows the descriptive statistics obtained for $L^{*} a^{*} b^{*}$ chromatic coordinates mean values within the different study groups over time. The initial moment is represented by $\mathrm{T}_{0}$, which corresponds to the baseline. Also, color variation $(\Delta \mathrm{E})$ between baseline and each of the subsequent color measurement periods of evaluation is shown in Table 2.

Table 2. Mean and standard deviation values of each of the $L^{*} a^{*} b^{*}$ coordinates of each experimental group for all periods of evaluation. $\Delta \mathrm{E}$ values express color variation from baseline $\left(\mathrm{T}_{0}\right)$ to each evaluation timepoint $\left(\mathrm{T}_{\mathrm{PO}}, \mathrm{T}_{72 \mathrm{H}}, \mathrm{T}_{7 \mathrm{D}}\right.$, and $\left.\mathrm{T}_{6 \mathrm{M}}\right)$.

\begin{tabular}{|c|c|c|c|c|c|c|c|}
\hline Groups & Coordinates & $\mathrm{T}_{0}$ & $\mathbf{T}_{\mathbf{P O}}$ & $\mathrm{T}_{72 \mathrm{H}}$ & $\mathrm{T}_{7 \mathrm{D}}$ & $\mathrm{T}_{6 \mathrm{M}}$ & $p$ \\
\hline \multirow{4}{*}{ Control/Saline } & $\mathrm{L}^{*}$ & $58.6 \pm 6.6$ & $61.6 \pm 6.0$ & $58.6 \pm 6.5$ & $60 \pm 6.6$ & $59.5 \pm 7.1$ & $=0.139$ \\
\hline & $a^{*}$ & $1 \pm 5.2$ & $2.8 \pm 5.2$ & $3.5 \pm 6.5$ & $3.2 \pm 5.9$ & $3.4 \pm 5.3$ & $=0.615$ \\
\hline & $b^{*}$ & $18.1 \pm 12.6$ & $17.6 \pm 11.7$ & $19.1 \pm 12.5$ & $19.3 \pm 12.3$ & $19 \pm 12.3$ & $=0.162$ \\
\hline & $\Delta \mathrm{E}$ & & $3.8 \pm 2.1$ & $2.0 \pm 0.7$ & $2.2 \pm 0.9$ & $1.6 \pm 0.6$ & $=0.615$ \\
\hline \multirow{4}{*}{ MTA/Saline } & $\mathrm{L}^{*}$ & $59.2 \pm 5.6$ & $60.4 \pm 5.6$ & $55.6 \pm 5.2$ & $52.3 \pm 5.1$ & $52.2 \pm 7.3$ & $<0.001^{\dagger}$ \\
\hline & $a^{*}$ & $2.0 \pm 2.4$ & $2.0 \pm 2.5$ & $1.1 \pm 1.6$ & $0.8 \pm 1.5$ & $0.7 \pm 1.1$ & $=0.070$ \\
\hline & $b^{*}$ & $15.6 \pm 6.5$ & $14.7 \pm 6.0$ & $12.0 \pm 5.3$ & $11.1 \pm 4.9$ & $11.3 \pm 5$ & $<0.001^{\dagger}$ \\
\hline & $\Delta \mathrm{E}$ & & $1.8 \pm 0.8$ & $5.5 \pm 1.1$ & $8.6 \pm 1.2$ & $8.5 \pm 2.6$ & $=0.001^{\dagger}$ \\
\hline \multirow{4}{*}{ Biodentine/Saline } & $\mathrm{L}^{*}$ & $55.6 \pm 4.9$ & $56.0 \pm 4.0$ & $55.6 \pm 4.4$ & $52.5 \pm 5.0$ & $55.5 \pm 4$ & $=0.012^{\dagger}$ \\
\hline & $a^{*}$ & $2.8 \pm 3$ & $3.2 \pm 2.8$ & $3.7 \pm 2.6$ & $3.3 \pm 2.7$ & $4.8 \pm 2.6$ & $<0.001^{+}$ \\
\hline & $b^{*}$ & $16.4 \pm 4.7$ & $16.6 \pm 4.1$ & $17.1 \pm 4.3$ & $16.2 \pm 3.8$ & $19 \pm 4.5$ & $=0.003^{+}$ \\
\hline & $\Delta \mathrm{E}$ & & $1.5 \pm 0.6$ & $1.4 \pm 0.7$ & $3.4 \pm 0.9$ & $3.4 \pm 0.9$ & $=0.006^{\dagger}$ \\
\hline \multirow{4}{*}{ TotalFill/Saline } & $\mathrm{L}^{*}$ & $60.1 \pm 5.4$ & $61.9 \pm 4.7$ & $61.2 \pm 5.2$ & $60.3 \pm 5.4$ & $60.1 \pm 4.7$ & $=0.162$ \\
\hline & $a^{*}$ & $0.8 \pm 2.7$ & $0.9 \pm 3.1$ & $1.1 \pm 2.9$ & $1.2 \pm 3.0$ & $1.9 \pm 3.0$ & $=0.011^{\dagger}$ \\
\hline & $b^{*}$ & $13.0 \pm 6.8$ & $14.2 \pm 6.8$ & $14.8 \pm 6.4$ & $14.6 \pm 6.6$ & $17.7 \pm 5.5$ & $=0.001^{\dagger}$ \\
\hline & $\Delta \mathrm{E}$ & & $2.3 \pm 0.9$ & $2.4 \pm 0.7$ & $2.2 \pm 0.5$ & $4.9 \pm 2.4$ & $=0.102$ \\
\hline \multirow{4}{*}{ PCM/Saline } & $\mathrm{L}^{*}$ & $59.2 \pm 3.4$ & $60.8 \pm 3.6$ & $60.7 \pm 3.6$ & $56.8 \pm 3.6$ & $61.6 \pm 1.3$ & $=0.003^{+}$ \\
\hline & $a^{*}$ & $1.4 \pm 3.1$ & $1.5 \pm 2.7$ & $1.2 \pm 2.9$ & $1.2 \pm 2.7$ & $0.1 \pm 2.5$ & $=0.171$ \\
\hline & $b^{*}$ & $15.1 \pm 6.5$ & $15.4 \pm 5.1$ & $15.7 \pm 6.3$ & $14.6 \pm 6.0$ & $14.4 \pm 5.3$ & $=0.139$ \\
\hline & $\Delta \mathrm{E}$ & & $2.5 \pm 0.5$ & $1.7 \pm 0.8$ & $2.6 \pm 1.5$ & $5.1 \pm 3.9$ & $=0.284$ \\
\hline
\end{tabular}


Table 2. Cont.

\begin{tabular}{|c|c|c|c|c|c|c|c|}
\hline Groups & Coordinates & $T_{0}$ & $\mathbf{T}_{\mathbf{P O}}$ & $\mathrm{T}_{72 \mathrm{H}}$ & $\mathrm{T}_{7 \mathrm{D}}$ & $\mathrm{T}_{6 \mathrm{M}}$ & $p$ \\
\hline \multirow{4}{*}{ Control/Blood } & $L^{*}$ & $58.4 \pm 9.2$ & $58.0 \pm 8.9$ & $53.5 \pm 12.0$ & $53.6 \pm 10.3$ & $51.3 \pm 7.9$ & $=0.053$ \\
\hline & $a^{*}$ & $1.0 \pm 3.2$ & $4.9 \pm 3.7$ & $3.9 \pm 4.0$ & $3.4 \pm 3.6$ & $3.3 \pm 2.8$ & $=0.034^{+}$ \\
\hline & $b^{*}$ & $12.5 \pm 5.9$ & $10.6 \pm 5.3$ & $7.5 \pm 4.8$ & $8.7 \pm 4.9$ & $10.8 \pm 5.1$ & $=0.022^{+}$ \\
\hline & $\Delta \mathrm{E}$ & & $4.4 \pm 1.4$ & $7.7 \pm 4$ & $6.7 \pm 2.8$ & $8.0 \pm 1.7$ & $=0.122$ \\
\hline \multirow{4}{*}{ MTA/Blood } & $\mathrm{L}^{*}$ & $58.4 \pm 5.1$ & $58.6 \pm 4.3$ & $54.5 \pm 4.1$ & $51.3 \pm 4.0$ & $52.1 \pm 5.1$ & $<0.001^{\dagger}$ \\
\hline & $a^{*}$ & $1.0 \pm 2.0$ & $1.5 \pm 1.6$ & $0.0 \pm 2.0$ & $-0.4 \pm 2.0$ & $0.6 \pm 1.9$ & $<0.001^{\dagger}$ \\
\hline & $b^{*}$ & $16.0 \pm 3.2$ & $13.5 \pm 4.3$ & $11.2 \pm 4$ & $9.7 \pm 3.8$ & $12.2 \pm 3.1$ & $<0.001^{\dagger}$ \\
\hline & $\Delta \mathrm{E}$ & & $3.4 \pm 2.7$ & $6.4 \pm 3.1$ & $9.8 \pm 2.6$ & $7.6 \pm 2.4$ & $=0.004^{+}$ \\
\hline \multirow{4}{*}{ Biodentine/Blood } & $L^{*}$ & $60.6 \pm 4.2$ & $60.6 \pm 4.0$ & $60.9 \pm 4.1$ & $57.3 \pm 3.9$ & $61.0 \pm 4.2$ & $=0.013^{\dagger}$ \\
\hline & $a^{*}$ & $0.8 \pm 2.4$ & $0.9 \pm 2.2$ & $1.4 \pm 2.5$ & $1.0 \pm 1.9$ & $1.9 \pm 2.3$ & $=0.010^{\dagger}$ \\
\hline & $b^{*}$ & $12.8 \pm 5.5$ & $12.2 \pm 4.8$ & $14.0 \pm 4.5$ & $12.4 \pm 3.8$ & $16.7 \pm 4.4$ & $=0.004^{\dagger}$ \\
\hline & $\Delta \mathrm{E}$ & & $1.6 \pm 1.4$ & $2.1 \pm 1.0$ & $3.8 \pm 1.2$ & $4.6 \pm 1.6$ & $=0.009^{\dagger}$ \\
\hline \multirow{4}{*}{ TotalFill/Blood } & $\mathrm{L}^{*}$ & $59.1 \pm 7.8$ & $59.4 \pm 7.0$ & $57.5 \pm 7.1$ & $54.6 \pm 6.5$ & $56.7 \pm 6.7$ & $=0.001^{\dagger}$ \\
\hline & $a^{*}$ & $0.5 \pm 2.5$ & $2.7 \pm 3.2$ & $1.7 \pm 3.0$ & $1.5 \pm 2.9$ & $1.8 \pm 2.9$ & $=0.015^{\dagger}$ \\
\hline & $b^{*}$ & $11.0 \pm 4.3$ & $10.3 \pm 4.5$ & $11.4 \pm 3.5$ & $10.2 \pm 3.3$ & $15.5 \pm 3.0$ & $=0.006^{\dagger}$ \\
\hline & $\Delta \mathrm{E}$ & & $3.1 \pm 2.1$ & $2.5 \pm 0.9$ & $5.1 \pm 1.3$ & $5.5 \pm 2.3$ & $=0.086$ \\
\hline \multirow{4}{*}{ PCM/Blood } & $L^{*}$ & $58.6 \pm 6.9$ & $58.6 \pm 4.8$ & $55.0 \pm 5.9$ & $51.0 \pm 5.6$ & $55.9 \pm 5.6$ & $=0.001^{\dagger}$ \\
\hline & $a^{*}$ & $2.0 \pm 2.2$ & $3.9 \pm 2.0$ & $4.8 \pm 3.7$ & $4.0 \pm 3.4$ & $3.2 \pm 2.6$ & $=0.004^{+}$ \\
\hline & $b^{*}$ & $15.9 \pm 6.8$ & $14.3 \pm 7.1$ & $15.8 \pm 4.9$ & $14.5 \pm 4.6$ & $18.6 \pm 5.6$ & $=0.003^{+}$ \\
\hline & $\Delta \mathrm{E}$ & & $3.3 \pm 1.2$ & $5.5 \pm 2.9$ & $8.4 \pm 3.0$ & $4.5 \pm 2.5$ & $=0.006^{\dagger}$ \\
\hline
\end{tabular}

$\mathrm{T}_{0}$ : baseline (after cavity preparation, before biomaterial placement); $\mathrm{T}_{\mathrm{PO}}$ : immediately after biomaterial placement and provisional restoration; $\mathrm{T}_{72 \mathrm{H}}$ : after $72 \mathrm{~h}$ of storage; $\mathrm{T}_{7 \mathrm{D}}$ : after 7 days of storage; and $\mathrm{T}_{6 \mathrm{M}}$ : after six months of storage. Mean \pm standard deviation. ${ }^{+}$Statistically significant difference $(p<0.05)$.

There are statistically significant differences $(\mathrm{F}(2.1192 .90)=33.58 ; p<0.001)$ regarding color variation $(\triangle \mathrm{E})$ over time. All CSC groups showed a statistically significant variation (mainly decreasing) in $L^{*}$ values over time, except TotalFill/Saline group. MTA presented a noticeable reduction in $L^{*}$ values from $\mathrm{T}_{\mathrm{PO}}$ to $\mathrm{T}_{7 \mathrm{D}}$, regardless of the treatment (saline or blood), as well as PCM contaminated by blood. However, at $\mathrm{T}_{6 \mathrm{M}}$, group comparison regarding lightness revealed no statistical differences between the tested biomaterials either when in contact with saline solution $(p=0.065)$ or blood $(p=0.102)$, which also applies to $\mathrm{a}^{*}$ and $\mathrm{b}^{*}$ values in both conditions (saline: $\mathrm{a}^{*} p=0.072 ; \mathrm{b}^{*} p=0.355$; blood: $\mathrm{a}^{*} p=0.148 ; \mathrm{b}^{*} p=0.060$ ). When in contact with saline solution, both MTA and Biodentine exhibited statistically significant color alteration over time ( $p=0.001$ and $p=0.012$, respectively). In the case of blood contamination, MTA kept presenting statistically significant color variation $(p=0.001)$ alongside Biodentine $(p=0.009)$, and PCM $(p=0.006)$. No statistically significant differences were detected regarding $\Delta \mathrm{E}$ of TotalFill groups over time, either in contact with saline solution $(p=0.102)$ or blood $(p=0.086)$. At 6 months all CSC groups presented perceptible color changes $(\Delta \mathrm{E}>3.3)$.

Statistical differences are found considering the factor material $(\mathrm{F}(8.45,92.90)=5.635 ; p<0.001)$, the factor treatment $(\mathrm{F}(2.11,92.90)=5.04 ; p=0.007)$ or both material and treatment $(\mathrm{F}(8.45,92.90)=3.31 ; p=0.002)$. The graphs on Figure 4 show the mean $\Delta \mathrm{E}$ over time, considering materials (Figure $4 a$ ) and treatment (Figure $4 b$ ) variation.

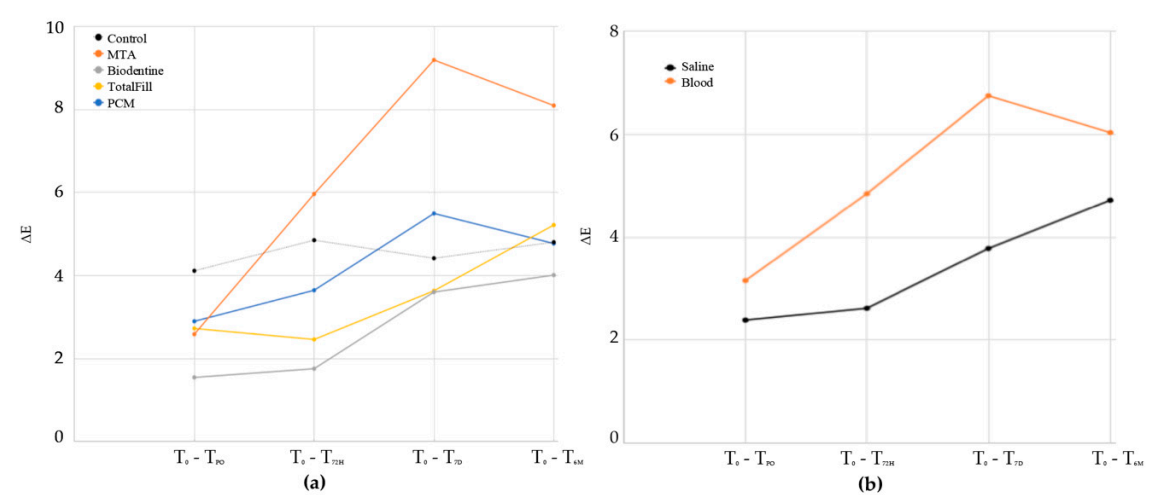

Figure 4. Color variation $(\triangle \mathrm{E})$ over time considering only the (a) materials (mineral trioxide aggregate (MTA), Biodentine, TotalFill, and PCM) and (b) treatment (Saline and Blood) variation. 
If solely the material is considered regardless the timing of evaluation, statistically significant differences are found $(\mathrm{F}(4,44)=13.05 ; p<0.001)$, which also applies to treatment $(\mathrm{F}(1,44)=23.11 ; p<0.001)$. MTA is the material showing lowest color stability, with statistically significant differences $(p<0.001)$ being detected in regard to all other tested CSCs (Biodentine, TotalFill, and PCM). Furthermore, no statistically significant differences are found between Biodentine, TotalFill, and PCM.

Additionally, there are statistically significant differences $(\mathrm{F}(4.44)=2.71 ; p=0.042)$ concerning the interaction between factor material and factor treatment. From all the CSC groups, MTA/Saline and MTA/Blood showed the highest color variation, with statistically significant differences. The total $\Delta \mathrm{E}$ from $\mathrm{T}_{0}$ to each of the following assessment periods is presented in Figure 5.

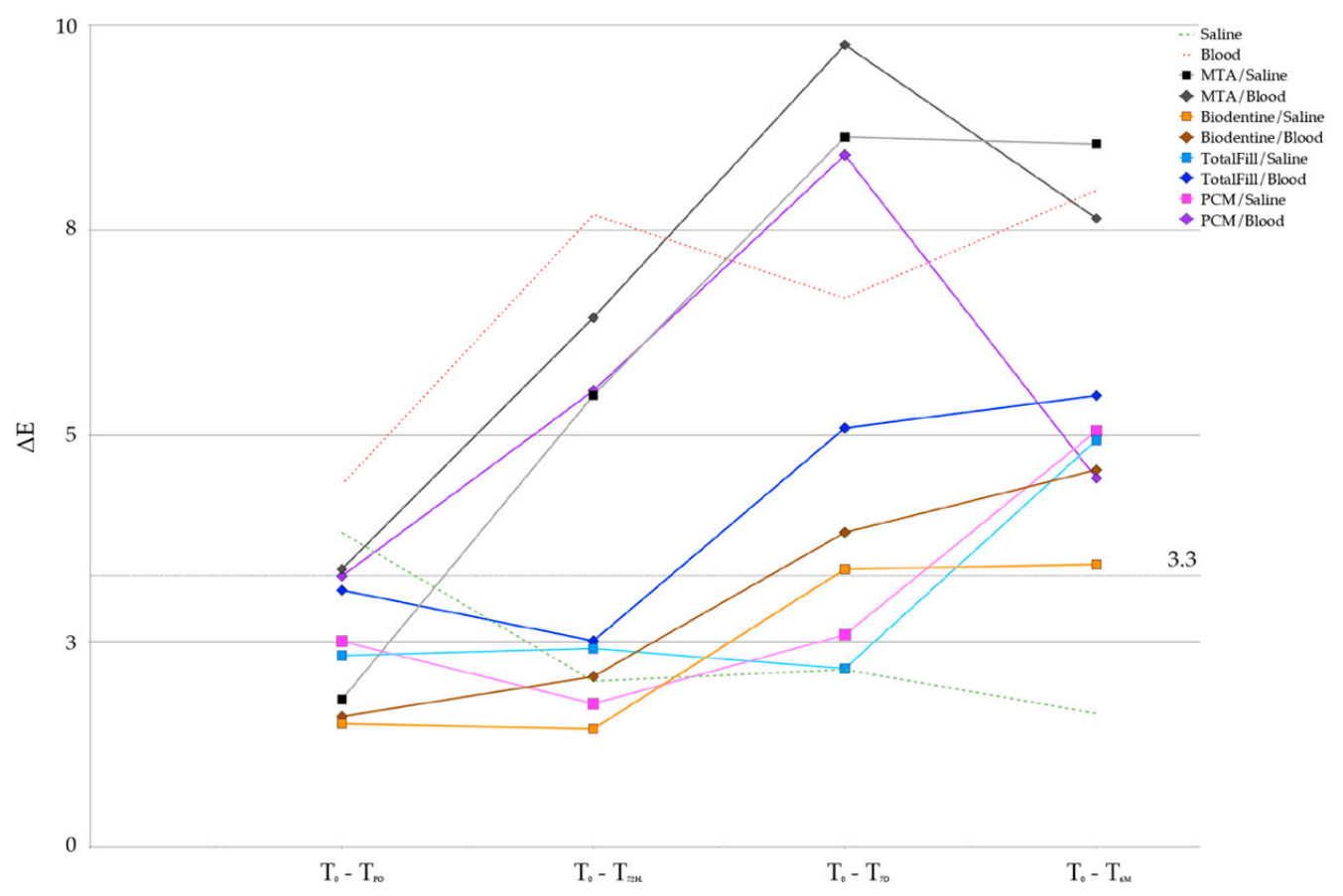

Figure 5. $\Delta \mathrm{E}$ of all experimental groups over time.

Table 3 is generated by performing group comparison according to individual evaluation of material/treatment interaction. PCM contaminated by blood led to a statistically higher color change when compared to Biodentine/Saline $(p=0.012)$.

Table 3. $p$-values obtained from group comparison regarding the interaction of factor material and factor treatment.

\begin{tabular}{|c|c|c|c|c|c|c|c|c|}
\hline MTA/Saline & Biodentine/Saline & TotalFill/Saline & PCM/Saline & Blood & MTA/Blood & Biodentine/Blood & TotalFill/Blood & PCM/Blood \\
\hline $0.010 *$ & 1.000 & 1.000 & 1.000 & 0.010 * & $0.001 *$ & 1.000 & 0.767 & 0.069 \\
\hline Biodentine/Saline & & 1.000 & 0.999 & $0.002 *$ & $<0.001 *$ & 0.999 & 0.544 & $0.012 *$ \\
\hline TotalFill/Saline & & & 1.000 & 0.010 * & $<0.001 *$ & 1.000 & 0.918 & 0.069 \\
\hline Blood & & & & & 1.000 & 0.012 * & 0.174 & 0.943 \\
\hline MTA/Blood & & & & & & $0.001 *$ & 0.028 * & 0.750 \\
\hline Biodentine/Blood & & & & & & & 0.939 & 0.082 \\
\hline TotalFill/Blood & & & & & & & & 0.739 \\
\hline
\end{tabular}

* Statistically significant difference $(p<0.05)$.

\section{Discussion}

The aim of the present ex vivo study was to evaluate and compare the discoloration potential of four different CSCs used in regenerative endodontic procedures, as well as to assess the role played by blood contamination on color variation severity when CSCs are applied. Theoretically, recently introduced biomaterials (Biodentine, TotalFill, and PCM) with a faster setting time when 
compared to the gold standard MTA might present superior color stability. Moreover, it is crucial to unveil the possible role played by blood when in contact with CSCs since blood exposure is likely to occur in both REPs and vital pulp therapy. An insight into these matters would allow adequate selection of the biomaterial to use based on the clinical conditions, ultimately allowing the accomplishment of better esthetic results.

In fact, it has been extensively reported that REPs lead to dental discoloration. This undesirable consequence of regenerative therapy is a major concern for both clinicians and patients, especially when an esthetic area is involved [4-7]. REPs protocol involves the creation of a cervical plug by placing a 3-4 mm CSC layer in the coronal portion of the root canal. Endodontic materials should present chromatic stability and optic properties similar to dental structures and not cause tooth discoloration over time [14]. However, as previously mentioned, the association of CSCs with color variation over time is well-described within the literature.

The obtained results show that there are statistically significant differences in $\Delta \mathrm{E}$ over time $(p<0.001)$. In fact, statistical differences were found considering the factor material $(p<0.001)$, the factor treatment $(p=0.007)$ or the interaction of both factors $(p=0.001)$, over time. Therefore, the null hypothesis, stating that there are no statistically significant differences between the experimental groups, has been rejected.

Several possible mechanisms have been outlined to explain color alteration following CSC placement, one being that the composition of the biomaterial is a major contributing factor to CSCs' discoloration potential [26]. MTA formula contains bismuth oxide as a radiopacifier $\left(\mathrm{Bi}_{2} \mathrm{O}_{3}\right)$, which has been proven to lead to tooth discoloration through its (1) reduction or (2) oxidation when in contact with strong oxidizing agents, such as dentin collagen and sodium hypochlorite, used as an irrigation solution [26]. In fact, previous studies consistently demonstrate MTA to exhibit lower color stability when compared to alternative calcium silicate-based cements [10,27], hypothesizing that the obtained results could arise from the incorporation of different radiopacifiers such as zirconium oxide and tantalum oxide, instead of bismuth oxide, in the more recently introduced CSCs [26].

Concerning blood contamination, the overall available scientific evidence shows blood exposure to be a factor that significantly exacerbates CSC color alteration [2]. This discoloration severity increase by blood might be related to material porosity and presence or absence of smear layer, which can reduce or increase dentin permeability, respectively. MTA has a longer setting time ( $2 \mathrm{~h}$ and $45 \mathrm{~min})$ compared with all the remaining three tested materials (Biodentine, $12 \mathrm{~min}$; TotalFill, $2 \mathrm{~h}$; PCM, $3 \mathrm{~min}$ ). Therefore, it seems MTA remains porous for longer which results in increased blood absorption and subsequent hemolysis, with consequently greater discoloration. On the other hand, a recent in vitro study found that contact with blood after a follow-up period of 6 months does not modify color alterations suffered by the biomaterials [24]. Our findings do not line up with the results of the previously mentioned study since, after a 6-month period of evaluation, the present study found statistically significant differences regarding the factor material, as well as regarding the treatment (blood/saline solution), meaning that blood exposure might be a critical factor in biomaterials' color variation. The contrasting results in respect to the role played by blood on color stability of CSCs, among studies with similar experimental protocols, might be related to the use of different experimental models. The study of Palma et al. [24] was conducted in an acrylic model, while the present study was made under ex vivo conditions. The use of natural human teeth may have led to a discoloration enhancement in the case of blood contamination due to the penetration of blood into the dentinal tubules [11]. For that reason the presence of blood has become a factor that significantly influences the chromatic result in the present study, which is in agreement with the overall literature [2]. It is, therefore, crucial to ensure complete hemostasis [26] and perform adequate blood clot stabilization, as well as to clean the blood present on dentin walls as meticulously as possible, to prevent blood influx into the tooth structure prior to CSC placement [24]. Moreover, the use of natural teeth as experimental model reproduces the clinical context more faithfully, thus allowing a better forecast of the impact of blood contamination on the esthetic outcome of the treatment. 
According to the results of the present study concerning material/treatment interaction, regardless of the treatment, MTA was the CSC showing the highest $\triangle \mathrm{E}$, which reflects that MTA presents the lowest color stability of all the tested biomaterials. Furthermore, MTA $\triangle \mathrm{E}$ values consistently indicate a noticeable color alteration regardless of the treatment, perceptible by the human eye $\Delta \mathrm{E} \geq 3.3$ ), therefore impairing the esthetic outcome of the procedure. Uesrichai et al. [7] assessed the clinical and radiographic outcome of partial pulpotomy using ProRoot MTA or Biodentine and found discoloration at follow-up (average 32 months) in both groups. Teeth treated with ProRoot MTA showed grey discoloration in $80 \%$ of the total sample and only $27 \%$ of teeth treated with Biodentine were affected. This difference between materials was statistically significant and supports the findings of the present research. Nowadays, Biodentine presents a viable alternative biomaterial to MTA. In addition to having zirconium oxide within its formulation, this CSC exhibits a shorter setting time. Previous ex vivo [11] and clinical [7] studies found greater color stability with Biodentine, which might be explained by the absence of bismuth oxide within its formulation and because of the faster setting time, which possibly limits blood sorption.

More recently, two different bismuth-free biomaterials were presented (TotalFill and PCM), with a shorter setting time when compared to MTA, which might be predictive of a favorable result concerning its color stability. While a favorable cytocompatibility profile of both biomaterials was reported in previous studies [28,29], there is no published data concerning color variation of PCM.

TotalFill was the only biomaterial which did not suffer statistically significant differences regarding $\Delta \mathrm{E}$ over time, either in contact with saline solution or blood. Since TotalFill is a pre-mixed material (ready-to-use), not subject to powder/water ratio variations, it appears that this material has less interaction with the environment (saline/blood), thus presenting lower color change in regard to the baseline. Although a previous clinical study [30] reported no differences between MTA and TotalFill regarding consequent tooth discoloration, the use of a double antibiotic intracanal paste which induces discoloration per se, a small sample size and the selected tooth color assessment methods, might explain the disagreement of the obtained results. The importance of in vitro and/or ex vivo studies that allow the evaluation of specific variables and avoid confounding factors is therefore highlighted. Although PCM is also a pre-dosed material (self-mixing syringe), statistically significant differences were verified in the PCM/Blood group over time, which looks to be associated with the different adhesion ability of both TotalFill and PCM to the surrounding dentine.

According to the results of the present study, TotalFill and PCM, like Biodentine, do not cause significant color change when compared with MTA. In fact, when individually analyzing the factor material, no statistically significant differences are found between Biodentine, TotalFill, and PCM. However, it is important to underline that, although TotalFill/Saline and TotalFill/Blood groups do not present statistically significant differences in terms of $\Delta \mathrm{E}$ over time, Biodentine presents absolute values of $\Delta \mathrm{E}$ consistently lower than those verified for TotalFill groups in both conditions (saline/blood), which might indicate that Biodentine leads to a lower clinical impact in terms of the perception of chromatic alterations. Moreover, group comparison of material/treatment interaction revealed that PCM contaminated by blood led to a statistically higher color change when compared to both Biodentine/Saline and Biodentine/Blood groups. Thus, considering the results of the present study, Biodentine might be the preferable material to use due to its lower esthetic impact.

To summarize, the choice of biomaterial presents a critical factor for the achievement of a successful esthetic outcome. According to our results over a six-month period, Biodentine is the material presenting the lowest discoloration potential, followed by TotalFill and PCM. MTA is the material with lowest color stability. These considerations are verified either in the presence or absence of blood during regenerative procedures. Furthermore, in addition to showing the lowest discoloration potential, it is noteworthy that previous studies demonstrate that Biodentine might allow the execution of bonding procedures directly over it in an immediate timeframe (12 min after biomaterial placement), therefore potentially maximizing the procedure's long-term success [31]. 
Regarding the limitations of the present study, the absence of positive pulp pressure inherent to an ex vivo model might limit the blood flow towards the periphery. Therefore, in the future, the role played by blood exposure should be confirmed in vivo. Moreover, a longer follow-up period is desirable to confirm the obtained results over a long-term perspective.

\section{Conclusions}

Within the limitations of the present study, our findings suggest that, after a six-month period of evaluation, blood exposure might be a critical factor in biomaterials color variation. Biodentine presents the lowest discoloration potential, followed by TotalFill and PCM, albeit without statistically significant differences. MTA exhibited the greatest color variation, consequently leading to a noticeable increase of tooth discoloration over time. The selection of the biomaterial should take into account the materials' discoloration potential.

Author Contributions: Conceptualization, P.J.P., J.A.M., J.C.R., R.I.F., and J.M.S.; methodology, P.J.P., R.I.F., J.A.M., J.C.R, and J.M.S.; software, F.C., R.I.F., P.J.P., J.A.M., D.S., P.D., and J.S.; validation, J.M.S. and P.J.P.; formal analysis, J.A.M., R.I.F., P.J.P., and J.S.; investigation, P.J.P., R.I.F., J.A.M., and J.S.; resources, J.M.S. and P.J.P.; data curation, J.A.M, D.S., P.D., J.S., and F.C.; writing-original draft preparation, J.S., J.A.M., P.J.P., and R.I.F.; writing—review and editing, P.J.P., J.A.M., and J.M.S.; visualization, R.I.F., J.A.M., J.S., and P.J.P.; supervision, P.J.P. and J.M.S.; project administration, J.S., J.A.M., D.S., and P.J.P.; funding acquisition, P.J.P., R.I.F, J.M.S., and J.C.R. All authors have read and agreed to the published version of the manuscript.

Funding: This research received no external funding.

Acknowledgments: The authors would also like to acknowledge Cláudia Brites of the Hard Tissues Histology Laboratory, Faculty of Medicine, University of Coimbra for assisting with the sample storage. The authors thank Dentsply Sirona, Septodont, FKG, and Coltène/Whaledent for graciously providing the experimental materials.

Conflicts of Interest: The authors declare no conflict of interest.

\section{References}

1. Diogenes, A.; Hargreaves, K.M. Microbial modulation of stem cells and future directions in regenerative endodontics. J. Endod. 2017, 43, S95-S101. [CrossRef] [PubMed]

2. Kahler, B.; Rossi-Fedele, G.; Chugal, N.; Lin, L.M. An evidence-based review of the efficacy of treatment approaches for immature permanent teeth with pulp necrosis. J. Endod. 2017, 43, 1052-1057. [CrossRef]

3. Palma, P.J.; Ramos, J.C.; Martins, J.B.; Diogenes, A.; Figueiredo, M.H.; Ferreira, P.; Viegas, C.; Santos, J.M. Histologic evaluation of regenerative endodontic procedures with the use of chitosan scaffolds in immature dog teeth with apical periodontitis. J. Endod. 2017, 43, 1279-1287. [CrossRef] [PubMed]

4. Nosrat, A.; Homayounfar, N.; Oloomi, K. Drawbacks and unfavorable outcomes of regenerative endodontic treatments of necrotic immature teeth: A literature review and report of a case. J. Endod. 2012, 38, 1428-1434. [CrossRef] [PubMed]

5. Kahler, B.; Mistry, S.; Moule, A.; Ringsmuth, A.K.; Case, P.; Thomson, A.; Holcombe, T. Revascularization outcomes: A prospective analysis of 16 consecutive cases. J. Endod. 2014, 40, 333-338. [CrossRef] [PubMed]

6. Kahler, B.; Rossi-Fedele, G. A review of tooth discoloration after regenerative endodontic therapy. J. Endod. 2016, 42, 563-569. [CrossRef]

7. Uesrichai, N.; Nirunsittirat, A.; Chuveera, P.; Srisuwan, T.; Sastraruji, T.; Chompu-Inwai, P. Partial pulpotomy with two bioactive cements in permanent teeth of 6- to 18-year-old patients with signs and symptoms indicative of irreversible pulpitis: A noninferiority randomized controlled trial. Int. Endod. J. 2019, 52, 749-759. [CrossRef]

8. Murray, P.E.; Garcia-Godoy, F; Hargreaves, K.M. Regenerative endodontics: A review of current status and a call for action. J. Endod. 2007, 33, 377-390. [CrossRef]

9. Diogenes, A.; Ruparel, N.B. Regenerative endodontic procedures: Clinical outcomes. Dent. Clin. North. Am. 2017, 61, 111-125. [CrossRef]

10. Keskin, C.; Demiryurek, E.O.; Ozyurek, T. Color stabilities of calcium silicate-based materials in contact with different irrigation solutions. J. Endod. 2015, 41, 409-411. [CrossRef] 
11. Ramos, J.C.; Palma, P.J.; Nascimento, R.; Caramelo, F.; Messias, A.; Vinagre, A.; Santos, J.M. 1-year in vitro evaluation of tooth discoloration induced by 2 calcium silicate-based cements. J. Endod. 2016, 42, 1403-1407. [CrossRef] [PubMed]

12. Lenherr, P.; Allgayer, N.; Weiger, R.; Filippi, A.; Attin, T.; Krastl, G. Tooth discoloration induced by endodontic materials: A laboratory study. Int. Endod. J. 2012, 45, 942-949. [CrossRef] [PubMed]

13. Krastl, G.; Allgayer, N.; Lenherr, P.; Filippi, A.; Taneja, P.; Weiger, R. Tooth discoloration induced by endodontic materials: A literature review. Dent. Traumatol. 2013, 29, 2-7. [CrossRef] [PubMed]

14. Camilleri, J. Color stability of white mineral trioxide aggregate in contact with hypochlorite solution. J. Endod. 2014, 40, 436-440. [CrossRef]

15. Palma, P.J.; Martins, J.; Diogo, P.; Sequeira, D.; Ramos, J.C.; Diogenes, A.; Santos, J.M. Does apical papilla survive and develop in apical periodontitis presence after regenerative endodontic procedures? Appl. Sci. 2019, 9, 3942. [CrossRef]

16. Parirokh, M.; Torabinejad, M. Mineral trioxide aggregate: A comprehensive literature review-Part III: Clinical applications, drawbacks, and mechanism of action. J. Endod. 2010, 36, 400-413. [CrossRef]

17. Kohli, M.R.; Yamaguchi, M.; Setzer, F.C.; Karabucak, B. Spectrophotometric analysis of coronal tooth discoloration induced by various bioceramic cements and other endodontic materials. J. Endod. 2015, 41, 1862-1866. [CrossRef]

18. Valles, M.; Roig, M.; Duran-Sindreu, F.; Martinez, S.; Mercade, M. Color stability of teeth restored with biodentine: A 6-month in vitro study. J. Endod. 2015, 41, 1157-1160. [CrossRef]

19. Marciano, M.A.; Duarte, M.A.; Camilleri, J. Dental discoloration caused by bismuth oxide in MTA in the presence of sodium hypochlorite. Clin. Oral Investig. 2015, 19, 2201-2209. [CrossRef]

20. Marciano, M.A.; Estrela, C.; Mondelli, R.F.; Ordinola-Zapata, R.; Duarte, M.A. Analysis of the color alteration and radiopacity promoted by bismuth oxide in calcium silicate cement. Braz. Oral Res. 2013, 27, 318-323. [CrossRef]

21. Marciano, M.A.; Costa, R.M.; Camilleri, J.; Mondelli, R.F.; Guimaraes, B.M.; Duarte, M.A. Assessment of color stability of white mineral trioxide aggregate angelus and bismuth oxide in contact with tooth structure. J. Endod. 2014, 40, 1235-1240. [CrossRef] [PubMed]

22. Felman, D.; Parashos, P. Coronal tooth discoloration and white mineral trioxide aggregate. J. Endod. 2013, 39, 484-487. [CrossRef] [PubMed]

23. Marin, P.D.; Bartold, P.M.; Heithersay, G.S. Tooth discoloration by blood: An in vitro histochemical study. Endod. Dent. Traumatol. 1997, 13, 132-138. [CrossRef] [PubMed]

24. Palma, P.J.; Marques, J.A.; Falacho, R.I.; Correia, E.; Vinagre, A.; Santos, J.M.; Ramos, J.C. Six-month color stability assessment of two calcium silicate-based cements used in regenerative endodontic procedures. J. Funct. Biomater. 2019, 10, 14. [CrossRef] [PubMed]

25. Marconyak, L.J., Jr.; Kirkpatrick, T.C.; Roberts, H.W.; Roberts, M.D.; Aparicio, A.; Himel, V.T.; Sabey, K.A. A Comparison of coronal tooth discoloration elicited by various endodontic reparative materials. J. Endod. 2016, 42, 470-473. [CrossRef]

26. Shokouhinejad, N.; Nekoofar, M.H.; Pirmoazen, S.; Shamshiri, A.R.; Dummer, P.M. Evaluation and comparison of occurrence of tooth discoloration after the application of various calcium silicate-based cements: An ex vivo study. J. Endod. 2016, 42, 140-144. [CrossRef]

27. Mozynska, J.; Metlerski, M.; Lipski, M.; Nowicka, A. Tooth discoloration induced by different calcium silicate-based cements: A systematic review of in vitro studies. J. Endod. 2017, 43, 1593-1601. [CrossRef]

28. Sequeira, D.B.; Seabra, C.M.; Palma, P.J.; Cardoso, A.L.; Peça, J.; Santos, J.M. Effects of a new bioceramic material on human apical papilla cells. J. Funct. Biomater. 2018, 9, 74. [CrossRef]

29. Koutroulis, A.; Kuehne, S.A.; Cooper, P.R.; Camilleri, J. The role of calcium ion release on biocompatibility and antimicrobial properties of hydraulic cements. Sci. Rep. 2019, 9, 19019. [CrossRef] 
30. Seirawan, M.Y.; Layous, K.; Seirawan, M.K.; Doumani, M. Coronal discoloration related to Bioceramic and Mineral Trioxide Aggregate coronal barrier in non-vital mature teeth undergoing regenerative endodontic procedures. World J. Dent. 2020, 11, 52-60.

31. Palma, P.J.; Marques, J.A.; Falacho, R.I.; Vinagre, A.; Santos, J.M.; Ramos, J.C. Does delayed restoration improve shear bond strength of different restorative protocols to calcium silicate-based cements? Materials 2018, 11, 2216. [CrossRef] [PubMed] 